\title{
Stochastic behaviors in plastic deformation of face-centered cubic micropillars governed by surface nucleation and truncated source operation
}

\author{
Ill Ryu ${ }^{*}$, Wei Cai ${ }^{b}$, William D. Nix ${ }^{c}$, Huajian Gao ${ }^{a}$ \\ ${ }^{\mathrm{a}}$ School of Engineering, Brown University, Providence RI, 02912, ${ }^{\mathrm{b}}$ Department of Mechanical Engineering, \\ 'Department of Materials Science and Engineering, Stanford University, Stanford CA, 94305-4040, United States. \\ *Corresponding author \\ E-mail address: ill_ryu@Brown.edu \\ Present address: 182 hope st. Barus \& Holley Bldg., Rm.737, Brown University, Providence, RI 02912 \\ Tel/Fax: +1 (650) 714-0731 / (401) 863-9025
}

\begin{abstract}
Three dimensional dislocation dynamics (DD) simulations are performed to investigate the governing mechanism of size dependent plastic deformation in submicron face-centered cubic (FCC) micropillars under uniaxial loading. Based on previous atomistic simulations, we introduce an algorithm for dislocation nucleation at the free surface as a function of stress and temperature in the $\boldsymbol{D D}$ simulation. The simulation results show stochastic behaviors in agreement with experimental observations, and reveal that dislocation nucleation at the free surface is the dominant mechanism of plastic flow in small pillars with diameters less than $200 \mathrm{~nm}$, while the operation of truncated dislocation sources is the governing mechanism in large pillars with diameters exceeding $1 \mu \mathrm{m}$. In between, both mechanisms come into play in a stochastic way.
\end{abstract}

Keywords: plasticity, micro-pillars, dislocation dynamics, dislocation nucleation 


\section{Introduction}

With the advent of micro-scale devices for engineering applications, mechanical properties of materials at small scales have attracted progressively more attention. Since it is now known that many mechanical properties at the sub-micron scale differ from those at the bulk scale, a fundamental understanding of such size dependence is required for further development.

Recent micromechanical experiments have revealed that the flow stress $(\tau)$ of metallic micropillars correlates with the pillar diameter $(d)$, with a scaling law of $\tau \propto d^{-0.5}$ to $d^{-1.0}$ for a wide variety of face centered cubic $(\boldsymbol{F C C})$ metals, even in the absence of strain gradients [1-7]. The reader is referred to the literature [810] for recent reviews on size effects in small structures. Among proposed models for these 'smaller is stronger' phenomena in metals, commonly accepted explanations include the dislocation starvation (DS) model $[11,12]$ and the single arm source $(\boldsymbol{S A S})$ model [13-18]. According to the $\boldsymbol{D S}$ model, smaller samples contain fewer dislocation sources making it easier for dislocations to escape, so that higher stresses are required to generate new dislocations for sustained plastic deformation. In the $\boldsymbol{S A S}$ model, the lengths of the truncated sources are shorter in smaller samples, so that higher stresses are required to operate them. Recent in-situ TEM observations provided support for both dislocation source exhaustion [19] and truncated source operation [20], and showed that both mechanisms could contribute to higher strengths in smaller volumes simultaneously rather than exclusively [21]. In both models, dislocation sources play a critical role in controlling the deformation behavior.

To achieve a better understanding of size dependent plasticity at the sub-micron scale, it is necessary to explore the details of dislocation behavior in small crystals. For this task, dislocation dynamics (DD) simulation provides a unique opportunity both to study the motion of individual dislocations, and to understand the mechanical behavior of materials in terms of the collective behavior of dislocations [17, 2229]. Fertig and Baker summarized the main features of widely used $\boldsymbol{D D}$ codes in their recent review [30]. Using $\boldsymbol{D D}$ simulations, Tang et al. showed that mobile dislocations in smaller micropillars could escape more quickly than in larger ones, so that the flow stress increases due to fewer sources [31, 32], thus providing a 
strong support for the $\boldsymbol{D S}$ model. On the other hand, it has also been shown that activation of truncated sources can successfully explain the size dependent flow behavior in both $\boldsymbol{F} \boldsymbol{C}$ and $\boldsymbol{B} \boldsymbol{C}$ crystals [18, 20, 33]. To model realistic situations, it is necessary to account for both internal sources from dislocation interaction and those in the vicinity of the free surface where dislocation nucleation is expected to occur. It is necessary to account for dislocation sources at the free surfaces especially in submicron crystals where the surface-tovolume ratio is very high. To our knowledge, most existing $\boldsymbol{D D}$ models consider only the internal sources while ignoring those associated with dislocation nucleation at the surface, and tend to underestimate the role of surface nucleation $[14,25,29,33,34]$.

With regard to external dislocation sources, the dislocation nucleation rate, a fundamental quantity of interest, has been calculated by continuum [35, 36] and atomistic models [37-41] . However, these approaches both have limitations. Continuum models are usually based on the linear elastic constitutive relation, which may not be valid under the high strain and nonlinear conditions of dislocation nucleation. On the other hand, direct molecular dynamics $(\boldsymbol{M D})$ simulations are limited to exceedingly high strain rates. To overcome these limitations, a recent approach is to combine reaction rate theories with atomistic models, wherein atomistic simulations are used to compute the activation barrier which then serves as an input parameter for the reaction rate theory to predict the dislocation nucleation rate $[42,43]$.

In this paper, we investigate the plastic flow behavior of single-crystal copper specimens under uniaxial loading using a dislocation dynamic model. In particular, attention will be given to the $\boldsymbol{F C C}$ metals in which perfect dislocations have a dominant role in the process of plastic deformation. We develop an algorithm within the $\boldsymbol{D D}$ framework to account for dislocation nucleation at the free surfaces, based on both atomistic modeling and reaction rate theory. Using this model, we explore its application to the plastic flow behavior of $\boldsymbol{F C} \boldsymbol{C}$ metal micropillars under uniaxial loading. Our $\boldsymbol{D D}$ simulation results, when compared to both experiments $[21,44,45]$ and theoretical models [13, 46] on micropillar deformation, show that exhaustion hardening and truncated source operation can play significant roles simultaneously .

\section{Simulation methods}




\subsection{Three dimensional dislocation dynamics in a cylinder}

Dislocation dynamics simulations were performed using a modified version of ParaDiS (Parallel Dislocation Simulator), a $\boldsymbol{D D}$ code originally developed at the Lawrence Livermore National Laboratory [22]. In ParaDiS, arbitrarily curved dislocations are discretized to a series of straight dislocation segments connected by nodes. To keep track of dislocation motion, we computed the force on each node based on the Peach-Koehler $(\boldsymbol{P K})$ formula. The local stress is determined from contributions from the applied loading, interactions with other dislocations, and corrections due to the existence of free surfaces. Once the force has been evaluated at each node, the dislocation velocity is computed using a mobility function [47]. In this work, we consider the over-damped regime in which the velocity of each dislocation is linearly proportional to the nodal force, subjected to glide plane constraints. Finally, dislocation movements are computed and updated with the consideration of topological changes and remesh requirements [22].

In order to model a micropillar compression experiment, the effect of the free surface on the stress field needs to be taken into account. To this end, various methods have been proposed to satisfy the tractionfree boundary condition on the free surface $[34,48,49]$. In this work, we consider the effect of the image stress using the Yoffe correction field of semi-infinite segments for computational efficiency, which has been shown to be a good approximation, especially for segments intersecting the surface $[50,51]$. To check the

validity of this approach, we compared a $\boldsymbol{D D}$ result using the Yoffe solution with the one using the spectral method [48], and did not observe a significant qualitative difference. Using a surface nucleation algorithm to be described in the next section in detail, a series of $\boldsymbol{D D}$ simulations of uniaxial tension were performed on $\boldsymbol{C u}$ single crystal micropillars with diameters ranging from $150 \mathrm{~nm}$ to $1000 \mathrm{~nm}$. For all the samples, the ratio of height to diameter is fixed at 5. The material properties and controlling parameters are listed in Table 1 .

\subsection{Dislocation nucleation at the free surface}

At the small scale of interest here, all possible dislocation sources need to be taken into account. Most existing $\boldsymbol{D D}$ models focus only on internal dislocation sources which mainly result from dislocationdislocation interactions, cross-slip or artificial pinning points [29, 52]. However, artificially created pinning 
points are not physically realistic and cross-slip does not occur easily in metals with low stacking fault energy. Internal dislocation sources from the interaction of dislocations can be naturally formed within the $\boldsymbol{D D}$ framework, while external sources due to dislocation nucleation at the free surface have not been carefully considered yet. Here, we develop a simple algorithm to implement surface nucleation in the ParaDiS cylinder code.

To accommodate dislocation sources associated with surface nucleation within the $\boldsymbol{D D}$ framework, we have adopted the nucleation rate from atomistic models [42, 43], usually expressed as a function of stress and temperature. The computed dislocation nucleation rate $(v)$ is expressed as

$$
v=v_{0} \exp \left(-\frac{Q(\sigma, T)}{k_{B} T}\right),
$$

where $v_{0}, k_{B} T$ are the attempt frequency, the thermal energy, respectively, and $Q$ is the activation free energy which is obtained from the previous study for dislocation nucleation from a $\boldsymbol{C} \boldsymbol{u}$ nanorod [42, 43]. Even though the nanorod considered in the atomistic model has a square cross section, which is different from the circular cross section considered here, the tabulated $Q(\sigma, \mathrm{T})$ function in [42] is used here given the various approximations already invoked in the present model. Furthermore, the previous work [42] considered nucleation of Shockley partial dislocations, while here we consider perfect dislocations, under the assumption that the trailing partial will quickly follow once the leading partial is nucleated. In this work, we focus on plastic flow at room temperature only, while noting that other temperatures could be treated in the same way. At given nucleation rate, we implement the surface dislocation nucleation in $\boldsymbol{D D}$ by adopting an algorithm commonly used in the kinetic Monte Carlo method [53], which can provide a simple yet powerful tool to obtain information about the statistical behavior of kinetics in many physical phenomena. To mimic the surface roughness, $N$ possible nucleation sites on the surface are randomly distributed on the surface with different stress concentration factors $(\boldsymbol{S C F})$, which follow a normal distribution with a mean of $\bar{\alpha}$, and a standard deviation of $\beta$. These values were chosen to fit the experimental values, as listed in Table 1. At a given stress and time, the nucleation probability for the $i^{\text {th }}$ site is simply computed as 


$$
P_{i}=v_{i} \times \Delta t=v_{0} \exp \left(-\frac{Q\left(\alpha_{i} \sigma, T\right)}{k_{B} T}\right) \times \Delta t,
$$

where $\alpha_{\mathrm{i}}$ is the stress concentration factor for the $i^{\text {th }}$ nucleation site. Note that activation energy for surface nucleation for each site varies due to different stress concentration factors. When the sum of all probability is larger than unity, the time step needs be adjusted by

$$
\Delta t=\frac{\Delta t_{0}}{\sum_{i=1}^{N} P_{i}}
$$

where $\Delta t_{0}$ is the original time step. The nucleation site is chosen stochastically, according to the nucleation probability, using the following relation;

$$
\sum_{i=1}^{m-1} P_{i} \leq \eta \sum_{j=1}^{N} P_{j}<\sum_{k=1}^{m} P_{k}
$$

where $i, j$, and $k$ are summation indices denoting the individual events, $m$ is the index of the chosen nucleation site, and $\eta$ is a random number uniformly distributed over the range $[0,1)[54]$. Once a nucleation site is chosen, we allow nucleation to occur only on the $1 / 2\langle 110\rangle /\{111\}$ type slip systems at the surface of the cylinder by creating dislocation half loops. The slip system with maximum $\boldsymbol{P K}$ force on the dislocation segment closest to the cylinder axis is selected for dislocation nucleation. When the magnitude of the maximum $\boldsymbol{P K}$ force is the same for multiple possible slip systems, the slip system of the half loop is randomly chosen among them, with the restriction that it moves inside the cylinder after nucleation. In addition, the initial radius of the newly-nucleated dislocation loop is kept as small as 50 times the magnitude of the Burgers vector. When a loop is formed at the surface, its contribution to the total plastic strain is added as follows

$$
\Delta \varepsilon_{i j}^{\text {plastic }}=\frac{\left(b_{i} n_{j}+b_{j} n_{i}\right)}{2 V} A_{0}
$$

where $b, n$ are the Burgers vector and slip normal, respectively; $V$ is the volume of the cylinder and $A_{0}$ is the initial area of the half loop. 


\subsection{Initial dislocation structure and loading mechanism}

At small scales, the mechanical response can be affected by the stability of the initial pinning points, so it is important to start the simulation with a reasonable initial dislocation structure [55]. To this end, several models have been suggested: a Frank network relaxed from randomly distributed straight and jogged dislocations [32], randomly distributed Frank-Read sources with pinning points [56] and randomly distributed loops with cross-slip allowed [26]. In this work, we adapt an approach similar to that by Motz et al.[26], in which an initial dislocation structure is prepared through the relaxation of randomly distributed dislocation glide loops in the absence of an applied stress until further dislocation activity is not noticeable. During the relaxation step, dislocation junctions were formed naturally without the aid of cross-slip, which in turn, formed internal dislocation sources. A detailed picture of the evolution of dislocation density and structure during the relaxation step can be seen in the Supplementary movie 1.

To be consistent with a stress-controlled experiment $[1,2]$ and existing DD studies $[14,32,33,52$, 57], external loading was imposed through a cut-off plastic strain rate method, where a constant increment of

$0.05 \mathrm{MPa}$ is applied at a cut-off plastic strain rate of $5 \times 10^{4} \mathrm{~s}^{-1}$ [58]. With these parameters, the simulations results are reasonably insensitive to the loading rate. The loading was applied along the cylinder axis, which has the [001] crystal orientation. Periodic boundary conditions are applied along the cylinder axis, and slip was allowed to occur only on $1 / 2\langle 110\rangle /\{111\}$ type slip systems.

\section{Simulation Results}

\subsection{Size-dependence of flow stress}

To investigate the effect of sample size, $\boldsymbol{D D}$ simulations were performed with different pillar diameters ranging from $150 \mathrm{~nm}$ to $1 \mu \mathrm{m}$. The initial dislocation density was about $10^{13} \mathrm{~m}^{-2}$. To obtain reasonable statistics, 10 independent simulations were performed under the same conditions. The simulation results indicate that the stress-strain curve clearly depends on the pillar diameter, as shown in Figure 1A. As the sample diameter decreases from $1 \mu \mathrm{m}$ to $150 \mathrm{~nm}$, the flow stress increases from roughly $300 \mathrm{MPa}$ to 1 
$G P a$. We could see stochastic variation in flow stress for all sized samples. The greatest variations were observed in the mid-sized sample of $600 \mathrm{~nm}$, with the highest flow stress reaching $900 \mathrm{MPa}$ and the lowest only $500 \mathrm{MPa}$. The evolving dislocation densities for differently sized pillars were calculated and plotted against strain in Figure 1B. Overall, the dislocation densities remain roughly constant with increasing plastic deformation after the initial yielding. For the largest samples with diameter of $1 \mu m$, dislocation densities seem to saturate with small fluctuations, while those among the smallest samples $(150,300 \mathrm{~nm}$ in diameter show significant fluctuations. Interestingly, the $600 \mathrm{~nm}$ samples showed both behaviors, i.e. with dislocation densities fluctuating in some cases but nearly saturating in others (See supplementary Figure S1).

These flow behaviors can be understood in terms of the microstructure of dislocations. For small samples, in the initial state of loading, dislocations move out of the sample easily, so a state lacking mobile dislocations is reached, consistent with the dislocation starvation model. When the stress reaches the critical stress for nucleation, dislocation half loops are generated from the surface, so that the flow stress reaches a plateau without hardening. Figure $2 A$ and Supplementary movie 2 show detailed stress-strain curves, dislocation density, and dislocation structure evolution for a typical result in a $150 \mathrm{~nm}$ sample. In all supplementary movies, the colors of the segments indicate the slip plane of the dislocations, among which the red segments indicate newly created dislocation junctions, formed through reactions of the type $1 / 2[01 \overline{1}]+1 / 2[\overline{1} 01]=1 / 2[\overline{1} 10]$ (Lomer lock). On the other hand, internal dislocation sources easily form in the larger samples (e.g., $1 \mu \mathrm{m}$ in diameter), so that the flow stress stops increasing once they start to operate. With a dislocation density of $10^{13} \mathrm{~m}^{-2}$, the pillar size of $1 \mu \mathrm{m}$ is not large enough and dislocation interactions are still insufficient to produce forest hardening, and the internal sources continue to operate. A detailed picture of the microstructure evolution with corresponding stress-strain curve and dislocation density for a 1 $\mu m$ diameter sample is provided in Figure $\mathbf{2 B}$ and Supplementary movie 3. In between, the $600 \mathrm{~nm}$ samples show both single arm source operation and surface nucleation in a stochastic way. For single arm operation, the dislocation density remains nearly constant with small fluctuations, while the surface nucleation results in larger fluctuations in dislocation density (See supplementary Figure S1).

\subsection{The effect of initial dislocation density}


To study the effect of the initial dislocation density, we have performed simulations with different initial densities ranging from $10^{13} \mathrm{~m}^{-2}$ to $10^{14} \mathrm{~m}^{-2}$. Stress-strain curves for three different initial dislocation densities are shown in Figure 3A-D. We see that the flow stress decreases with increasing initial dislocation density for all sample sizes, contrary to the conventional Taylor hardening relationship. It can be understood from the fact that dislocations now act as "carriers of plasticity", rather than "obstacles to dislocation motion" as in the forest hardening mechanism. For the $150 \mathrm{~nm}$ diameter samples, dislocations are so scarce that all mobile dislocations move out of the samples in the initial stage of loading. For further deformation, the stress needs to reach a level that brings external sources associated with surface nucleation of dislocations into play. As a result, the flow stress can always reach the nucleation stress without noticeable dislocation interactions. Due to the various stress concentration factors of the nucleation sites, the flow stresses exhibit stochastic behavior, even with the same initial dislocation densities, as shown in Figure 3A. For the intermediate sized samples (300, $600 \mathrm{~nm}$ in diameter), we see both truncated source operation and surface nucleation, resulting in more predominant stochastic variations in the flow stress (Figure 3B, 3C). Since it is more probable for dislocations to interact with each other at higher initial densities, the higher the initial dislocation density, the smaller the pillar size at which highly stochastic flow behavior is observed. For example, at a low initial dislocation density of $10^{13} \mathrm{~m}^{-2}$, the most stochastic flow behavior is observed for $600 \mathrm{~nm}$ and above samples, whereas it occurs for $300 \mathrm{~nm}$ and above samples at a higher initial dislocation density of $10^{14} \mathrm{~m}^{-2}$. In contrast,

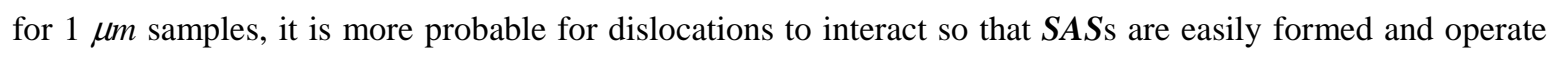
almost continuously. Due to computational limitation, we could not perform $\boldsymbol{D D}$ simulations for the cases with the diameter of $1 \mu \mathrm{m}$ and the initial dislocation density of $10^{14} \mathrm{~m}^{-2}$ (Figure $\left.3 \boldsymbol{D}\right)$ ). However, the initial dislocation density would not give rise to noticeable difference in the flow behavior at the sample size of 600 $n m$, when the initial density was higher than $5 \times 10^{13} \mathrm{~m}^{-2}$ (Figure $3 C$ ). Flow behaviors in $1 \mu \mathrm{m}$ samples are highly expected to show similar trends. In addition, the fluctuations between large samples with different initial dislocation densities are not as large as those between small samples, so that the flow stresses are quite low for the largest sample. 
To see the size dependence clearly, the flow stresses at the plateau were determined and plotted against the corresponding pillar diameters in Figure 4. DD results clearly show size dependent plastic flow behavior, but the flow stresses from $\boldsymbol{D} \boldsymbol{D}$ results were somewhat higher than experimental results. It is expected that other types of defects such as impurities and point defects could act as additional dislocation sources, so that the plastic flow would occur at lower stress in experiments. However, the log-log plot gives a size dependence exponent of about 0.73 , which is consistent with experimentally observed exponents for FCC metal pillars [21, 44, 45]. Experimental details are summarized in Table 2.

\section{Comparison with theoretical models for both surface nucleation (SN) and single arm source (SAS) models}

To have a quantitative understanding of the governing mechanism for plasticity at the submicron scale, we compared our $\boldsymbol{D D}$ results with existing theoretical models for both truncated source operation and surface nucleation.

\subsection{Surface nucleation model}

We start by considering the kinetic nucleation model by Nix and Lee [46], which envisages surface nucleation as a controlling mechanism for micropillar plasticity. In this model, mobile dislocation density is controlled by a competition between the rate of dislocation nucleation at the surface and the rate of annihilation out of the free surface, so that structure evolution law is simply

$$
\dot{\rho}=\dot{\rho}^{+}-\dot{\rho}^{-}
$$

where $\dot{\rho}^{+}$stands for the rate of increase in dislocation density from surface nucleation, and $\dot{\rho}^{+}$denotes the annihilation rate. Now, we may express the increasing rate associated with nucleation as follows:

$$
\dot{\rho}^{+}=\left(v_{0} \exp \left(\frac{-\Delta G(\sigma, T)}{k_{B} T}\right)\right) \times\left(\frac{\pi D h}{b^{2}}\right) \times\left(\frac{\pi / 4 D}{h \frac{\pi D^{2}}{4}}\right),
$$

where the first term stands for the rate of nucleation per site, the second term represents the maximum number of possible nucleation sites at the surface and the last terms denotes the average dislocation length per unit 
volume. Here, we modified the original expression by taking the nucleation rate from the atomistic simulation results [43]. The effect of stress concentration is ignored in this simple analysis. For the depletion rate, it is assumed that dislocations can travel across the diameter of the pillar during their lifetime, which is then computed by dividing the pillar diameter by the dislocation velocity. Then, the depletion rate is simply computed by dividing the mobile dislocation density by the lifetime of traveling dislocations as follows

$$
\dot{\rho}^{-}=\frac{\rho}{t_{l i f e}}=\rho \frac{v_{d}}{D}=\rho \frac{M S \sigma b}{D}
$$

where $v_{d}, M$ and $\mathrm{S}$ denote the dislocation velocity, mobility and Schmid factor. To compute the rate of depletion, we take the dislocation density from $\boldsymbol{D D}$ results when flow stress reaches a plateau at each case. In

Figure 5, we plot these two rates as a function of the applied stress for different sample sizes. Here, the initial dislocation density in the $\boldsymbol{D D}$ model is $10^{13} \mathrm{~m}^{-2}$; we could not see noticeable quantitative differences with different initial dislocation densities. It is worth noting that, in equation 7 , the dislocation nucleation rate is independent of the pillar diameter [46]. From the form of equation 6, we computed the flow stress for the steady condition at which the rate of dislocation nucleation is balanced by the rate of depletion out of the surface and compared the results with our $\boldsymbol{D D}$ results, as shown in Figure 6. We can see that the size dependence from the surface nucleation model is not very significant. Therefore, we conclude that surface nucleation provides a maximum stress limit for the flow stress.

\subsection{Single arm source model}

The critical resolved shear stress for $\boldsymbol{S A S}$ operation has been computed in various papers [13, 16, 59, 60]. Among them, we take Parthasarathy et al.'s $\boldsymbol{S A S}$ model [13] with input from our $\boldsymbol{D D}$ results. The critical resolved stress is expressed as

$$
\sigma_{c r}^{S A S}=\sigma_{0}+\frac{1}{2} \mu b \sqrt{\rho}+\frac{\alpha \mu b}{\lambda_{c r}}
$$

where the first term indicates the friction stress [61], the second term represents the internal stresses associated with dislocations, and the third term represents the resistance associated with line tension. Here $\alpha$ is a constant and $\lambda_{c r}$ is the statistical average length of the weakest single arm dislocation source, which is 
measured from our $\boldsymbol{D D}$ results. To calculate it from the $\boldsymbol{D D}$ results, we look for the first formed single or doubly connected arms and compute the minimum length from the surface in its slip plane. Recently, Cui et al. [33] reported that the critical source length calculated from $\boldsymbol{D D}$ is linearly proportional to the sample diameter. Using our DD results we get the same trend as shown in Figure 7, where we have plotted the results for different initial dislocation densities. With the aid of these $\boldsymbol{D D}$ results, the flow stress is calculated and added in Figure 6. It exhibits clear size dependence and agrees well with our $\boldsymbol{D D}$ results.

\section{Discussion}

For submicron FCC micro pillars, it has been postulated that the plastic deformation can be divided into three regimes [9, 52]. For small samples with diameters less than $150 \mathrm{~nm}$, the initial dislocations hardly interact with each other before they are driven out of the sample, so that further plastic deformation is governed by dislocation nucleation at the surface. For large samples with diameters exceeding $1000 \mathrm{~nm}$, since it is almost impossible to avoid dislocation interactions, $\boldsymbol{S A S}$ s are formed under the loading and the operation of these $\boldsymbol{S A S}$ s is believed to be the governing mechanism. In between these two regimes, both $\boldsymbol{S N}$ and $\boldsymbol{S A S}$ are likely to occur simultaneously. In this work, for the qualitative characterization of these regimes, we have extended the existing $\boldsymbol{D D}$ models by implementing a surface nucleation scheme. To see the governing mechanisms for plasticity with respect to the sample size and initial dislocation density, we have investigated the evolution of the dislocation microstructures, from which we could determine which mechanism was responsible for the plastic flow behavior under a given set of conditions: if a single arm source operates at the plateau in the stress-strain curve, the operation of $\boldsymbol{S A S}$ is taken to be the governing mechanism, while the $\boldsymbol{S N}$ is the governing plastic flow mechanism when dislocation nucleation is observed predominantly. As a result, $S N$ is observed to be the controlling mechanism for the smallest samples (150 $\mathrm{nm}$ diameter samples), while $\boldsymbol{S A S}$ always form in the largest samples (1000 $\mathrm{nm}$ diameter samples). As expected, the behavior at intermediate pillar sizes depends on the initial dislocation density. Since it is more probable for dislocations to interact at higher initial dislocation densities, the $\boldsymbol{S A S}$ mechanism extends to smaller sizes at higher densities.

Compared to bulk $\boldsymbol{F C C}$ metals, where the flow stress increases with increasing dislocation density due to forest hardening, the results of our $\boldsymbol{D D}$ simulations for micropillars exhibit lower flow stresses with 
increasing initial dislocation density. This is consistent with the findings of Lee et al. [62] who showed that pre-straining of $\boldsymbol{A u}$ pillars dramatically reduces their flow strength. It suggests that dislocations in micropillars are often carriers of plasticity [63], rather than obstacles to dislocation motion assumed in the Taylor hardening relation.

As reported previously based on experiments and other $\boldsymbol{D D}$ results, stochastic jerky flow behavior is frequently observed in stress-strain relations in nanopillars. Interestingly, the largest variations in flow stress are observed for intermediate sized samples, rather than for the smallest pillars in our $\boldsymbol{D D}$ simulations. This is caused by the fact that stochastic variation from both surface nucleation and single arm operation would come into play in the intermediate size in our models. However, in this work, we have ignored other causes of stochastic behavior in the flow stress such as impurities, surface steps. To model realistic stochastic flow behavior, further research of other dislocation sources is needed.

The plastic deformation from both mechanisms is predicted to be highly localized from $\boldsymbol{D} \boldsymbol{D}$ results. In the surface nucleation mechanism, dislocation nucleation occurs only at the selective nucleation sites which have highest stress concentration factors, because the nucleation rate is highly sensitive to the stress. In the single arm source mechanism, the truncated Frank-Read sources can operate for many cycles without being shut down due to lack of obstacles on its glide plane. However, the present analysis is still questionable regarding the immortality of the sources in both mechanisms. Atomistic modeling and experimental observations showed that dislocation nucleation at the surface could occur throughout whole sample, rather than at only a few preferential sites [64-66]. In addition, recent atomistic modeling showed that the pinning point in the form of a Lomer-Cottrell $(\boldsymbol{L C})$ jog could be destroyed by the rotation of the jog on its $\{100\}$ glide plane, even in the absence of the influence of other dislocations [67]. In our $\boldsymbol{D D}$ model, we assume that only octahedral glide occurs without considering other possible pinning point destruction processes, including either other slip systems or climb, so that we overestimate the stability of these pinning points. While our investigations provides some insight into the governing mechanisms for deformation at small strains, the scope of its application to the high strain regime may be limited by the lack of the knowledge of the stability 
of the dislocation sources. To this end, careful consideration of the stability of truncated source operation is needed for realistic modeling of the high strain regime.

In the $\boldsymbol{D D}$ simulations, intermittent jerky plastic flow is evident in the stress-strain curves, indicating cooperation of both $\boldsymbol{S A S}$ operation and $\boldsymbol{S N}$ mechanisms as the dislocation density shows large fluctuations with high frequency. In addition, the flow stress eventually converges to a constant level in the absence of strain hardening, as already shown in previous $\boldsymbol{D D}$ results [33]. For small samples at which $\boldsymbol{S N}$ is the dominant mechanism, nucleation occurs explosively at the critical stress so that the flow stress does not increase further because of the large amount of plastic deformation triggered by the nucleation events. For large enough samples where the $\boldsymbol{S A S}$ operates, the single armed or doubly connected sources are stable due to the absence of dislocation interactions at this still small scale. Since the stability of the internal dislocation sources strongly depends on the dislocation reactions, strain hardening would be expected for very large pillar sizes and very high dislocation densities, involving various dislocation interactions such as dipoles, junctions, and Frank-Read sources.

\section{Conclusions}

Three dimensional $\boldsymbol{D D}$ simulations have been performed in order to investigate the governing mechanisms of size dependent plasticity in submicron pillars. Our $\boldsymbol{D D}$ simulations, which include a surface nucleation scheme, show that the flow stress increases with decreasing pillar size and decreasing initial dislocation density. In addition, $\mathbf{S N}$ is found to be the dominant mechanism in pillars with diameters less than $200 \mathrm{~nm}$, while $\boldsymbol{S A S}$ operation is the governing mechanism for plastic flow in pillars larger than $1 \mu \mathrm{m}$. In between, both mechanisms occur in a stochastic way. We also compared our $\boldsymbol{D D}$ results with continuum models for $\boldsymbol{S A S}$ and $\boldsymbol{S N}$ and showed that $\boldsymbol{S N}$ limits the flow stress with small size dependence, while $\boldsymbol{S A S}$ operation provides strong size dependence mainly for large sized pillars. Results from both the $\boldsymbol{D} \boldsymbol{D}$ model and theoretical models are in good agreement with many experiments on $\boldsymbol{F C C}$ metal pillars.

\section{Acknowledgements}


The authors gratefully acknowledge funding from the US Department of Energy through the DOE EPSCoR Implementation grant no. DE-SC0007074 and the GM-Brown Collaborative Research Laboratory on Computational Materials Science. W.C. and W.D.N. gratefully acknowledges support from the Office of Science, Office of Basic Energy Sciences, of the U.S. Department of Energy under Contract No. DESC0010412 and No. DE-FG02-04ER46163, respectively. We would like to thank Prof. S.W. Lee of University of Connecticut for useful discussions. 


\section{References}

[1] Uchic MD, Dimiduk DM, Florando JN, Nix WD. Science 2004;306:1134.

[2] Dimiduk DM, Uchic MD, Parthasarathy TA. Acta Mater 2005;53:4065.

[3] Greer JR, Oliver WC, Nix WD. Acta Mater 2005;53:1821.

[4] Dimiduk DM, Woodward C, LeSar R, Uchic MD. Science 2006;312:1188.

[5] Kiener D, Motz C, Schoberl T, Jenko M, Dehm G. Adv Eng Mater 2006;8:1119.

[6] Volkert CA, Lilleodden ET. Philos Mag 2006;86:5567.

[7] Ng KS, Ngan AHW. Acta Mater 2008;56:1712.

[8] Greer JR, De Hosson JTM. Prog Mater Sci 2011;56:654.

[9] Kraft O, Gruber PA, Monig R, Weygand D. Annual Review of Materials Research, Vol 40 2010;40:293.

[10] Uchic MD, Shade PA, Dimiduk DM. Annu Rev Mater Res 2009;39:361.

[11] Greer JR, Nix WD. Phys Rev B 2006;73.

[12] Nix WD, Greer JR, Feng G, Lilleodden ET. Thin Solid Films 2007;515:3152.

[13] Parthasarathy TA, Rao SI, Dimiduk DM, Uchic MD, Trinkle DR. Scripta Mater 2007;56:313.

[14] Rao SI, Dimiduk DM, Parthasarathy TA, Uchic MD, Tang M, Woodward C. Acta Mater 2008;56:3245.

[15] Rao SI, Dimiduk DM, Tang M, Parthasarathy TA, Uchic MD, Woodward C. Philos Mag 2007;87:4777.

[16] Ng KS, Ngan AHW. Scripta Mater 2008;59:796.

[17] El-Awady JA, Wen M, Ghoniem NM. J Mech Phys Solids 2009;57:32.

[18] Mompiou F, Legros M, Sedlmayr A, Gianola DS, Caillard D, Kraft O. Acta Mater 2012;60:977.

[19] Shan ZW, Mishra RK, Asif SAS, Warren OL, Minor AM. Nat Mater 2008;7:115.

[20] Oh SH, Legros M, Kiener D, Dehm G. Nat Mater 2009;8:95.

[21] Kiener D, Minor AM. Nano Lett 2011;11:3816.

[22] Arsenlis A, Cai W, Tang M, Rhee M, Oppelstrup T, Hommes G, Pierce TG, Bulatov VV. Model Simul Mater Sc 2007;15:553.

[23] Devincre B, Kubin LP. Mat Sci Eng a-Struct 1997;234:8.

[24] Espinosa HD, Panico M, Berbenni S, Schwarz KW. Int J Plasticity 2006;22:2091.

[25] Liu ZL, Liu XM, Zhuang Z, You XC. Scripta Mater 2009;60:594.

[26] Motz C, Weygand D, Senger J, Gumbsch P. Acta Mater 2009;57:1744.

[27] Schwarz KW. J Appl Phys 1999;85:120.

[28] Weygand D, Friedman LH, van der Giessen E, Needleman A. Mat Sci Eng a-Struct 2001;309:420.

[29] Zhou CZ, Biner SB, LeSar R. Acta Mater 2010;58:1565.

[30] Fertig RS, Baker SP. Prog Mater Sci 2009;54:874.

[31] Tang H, Schwarz KW, Espinosa HD. Acta Mater 2007;55:1607.

[32] Tang H, Schwarz KW, Espinosa HD. Phys Rev Lett 2008;100.

[33] Cui YN, Lin P, Liu ZL, Zhuang Z. Int J Plasticity 2014;55:279.

[34] Weygand D, Poignant M, Gumbsch P, Kraft O. Mat Sci Eng a-Struct 2008;483-84:188.

[35] Xu G, Argon AS, Oritz M. Philos Mag A 1997;75:341.

[36] Aubry S, Kang K, Ryu S, Cai W. Scripta Mater 2011;64:1043.

[37] Tschopp MA, Spearot DE, McDowell DL. Model Simul Mater Sc 2007;15:693. 
[38] Bringa M, Rosolankova $\mathrm{K}$, Rudd RE, Remington BA, Wark JS, Duchaineau M, Kalantar H, Hawreliak J, Belak J. Nat Mater 2006;5:805.

[39] Zhu T, Li J, Samanta A, Leach A, Gall K. Phys Rev Lett 2008;100.

[40] Diao J, Gall K, Dunn ML. Nano Lett 2004;4:1863.

[41] Rabkin E, Nam HS, Srolovitz DJ. Acta Mater 2007;55:2085.

[42] Ryu S, Kang K, Cai W. J Mater Res 2011;26:2335.

[43] Ryu S, Kang K, Cai W. Proceedings of the National Academy of Sciences 2011;108:5174.

[44] Jennings AT, Burek MJ, Greer JR. Phys Rev Lett 2010;104.

[45] Kiener D, Minor AM. Acta Mater 2011;59:1328.

[46] Nix WD, Lee SW. Philos Mag 2011;91:1084.

[47] Cai W, Bulatov VV. Mat Sci Eng a-Struct 2004;387:277.

[48] Weinberger CR, Cai W. J Mech Phys Solids 2007;55:2027.

[49] El-Awady JA, Biner SB, Ghoniem NM. J Mech Phys Solids 2008;56:2019.

[50] Yoffe EH. Philos Mag 1961;6:1147.

[51] Weinberger CR, Aubry S, Lee SW, Nix WD, Cai W. Model Simul Mater Sc 2009;17.

[52] Zhou C, Beyerlein IJ, LeSar R. Acta Mater 2011;59:7673.

[53] Bortz AB, Kalos MH, Lebowitz JL. Journal of Computational Physics 1975;17:10.

[54] Battaile CC. Computer Methods in Applied Mechanics and Engineering 2008;197:3386.

[55] Senger J, Weygand D, Motz C, Gumbsch P, Kraft O. Acta Mater 2011;59:2937.

[56] Senger J, Weygand D, Motz C, Gumbsch P, Kraft O. Philos Mag 2010;90:617.

[57] El-Awady JA, Rao SI, Woodward C, Dimiduk DM, Uchic MD. Int J Plasticity 2011;27:372.

[58] Lee S-W, Jennings AT, Greer JR. Acta Mater 2013;61:1872.

[59] Norfleet DM, Dimiduk DM, Polasik SJ, Uchic MD, Mills MJ. Acta Mater 2008;56:2988.

[60] Lee SW, Nix WD. Philos Mag 2012;92:1238.

[61] Liu Y, Varghese S, Ma J, Yoshino M, Lu H, Komanduri R. Int J Plasticity 2008;24:1990.

[62] Lee SW, Han SM, Nix WD. Acta Mater 2009;57:4404.

[63] Johnston WG, Gilman JJ. J Appl Phys 1959;30:129.

[64] Wu ZX, Zhang YW, Jhon MH, Greer JR, Srolovitz DJ. Acta Mater 2013;61:1831.

[65] Wu ZX, Zhang YW, Jhon MH, Gao HJ, Srolovitz DJ. Nano Lett 2012;12:910.

[66] Kiener D, Grosinger W, Dehm G, Pippan R. Acta Mater 2008;56:580.

[67] Weinberger CR, Cai W. Scripta Mater 2011;64:529. 


\section{Figure captions}

Figure1. (a) Stress vs. strain curves (b) dislocation density evolution for the $150 \mathrm{~nm}, 300$ $\mathrm{nm}, 600 \mathrm{~nm}$ and $1000 \mathrm{~nm}$ sized pillars with the initial dislocation density of $10^{13} \mathrm{~m}^{-2}$. For each size pillar, dislocation density is plotted after averaging them within data bins. The symmetric error bars denote the standard deviation from the mean values.

Figure2. Stress and density evolution under the loading for (a) a $150 \mathrm{~nm}$ (b) $1000 \mathrm{~nm}$ sized pillar. Inset denotes dislocation structures with respect to points along the stress-strain curve.

Figure3. Stress vs. strain curves with different initial dislocation densities for (a) $150 \mathrm{~nm}$, (b) $300 \mathrm{~nm}$, (c) $600 \mathrm{~nm}$ and (d) $1000 \mathrm{~nm}$ sized pillars.

Figure4. (a) Comparison between flow stress from micro-pillar experiments and $\boldsymbol{D} \boldsymbol{D}$ results at steady flow state. (b) Log-log plot.

Figure5. Rate of increasing in dislocation density and the depletion rate with respect to the applied stress. The initial dislocation density is $10^{13} \mathrm{~m}^{-2}$.

Figure6. Comparison between $\boldsymbol{D D}$ results and theoretical models of $\boldsymbol{S A S}$ and $\boldsymbol{S N}$ mechanisms with the initial density of $10^{13} \mathrm{~m}^{-2}$.

Figure7. Critical length of initial $\boldsymbol{S A S}$ normalized by the pillar diameter. 


\section{Figure(s)}

Figure 1A

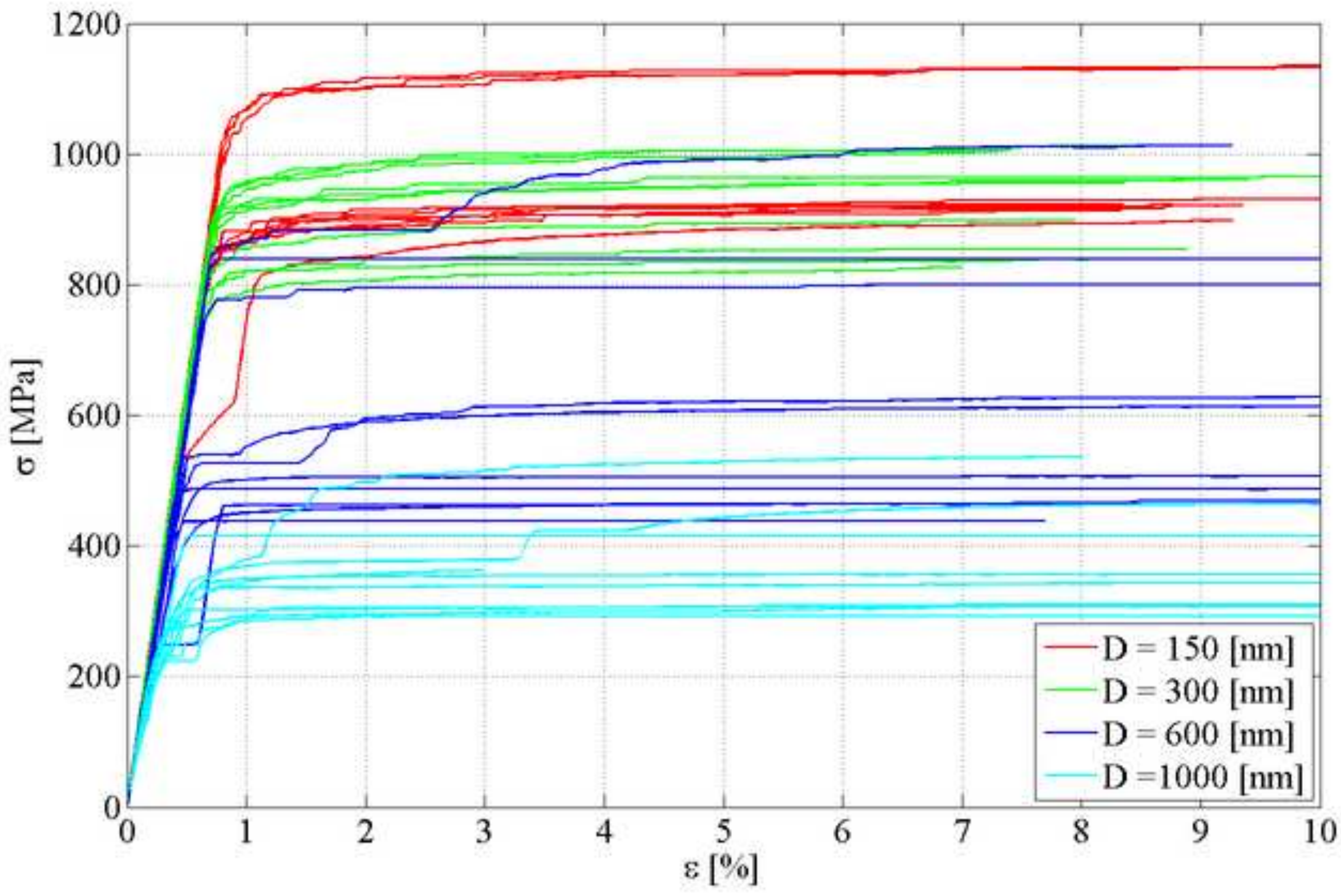




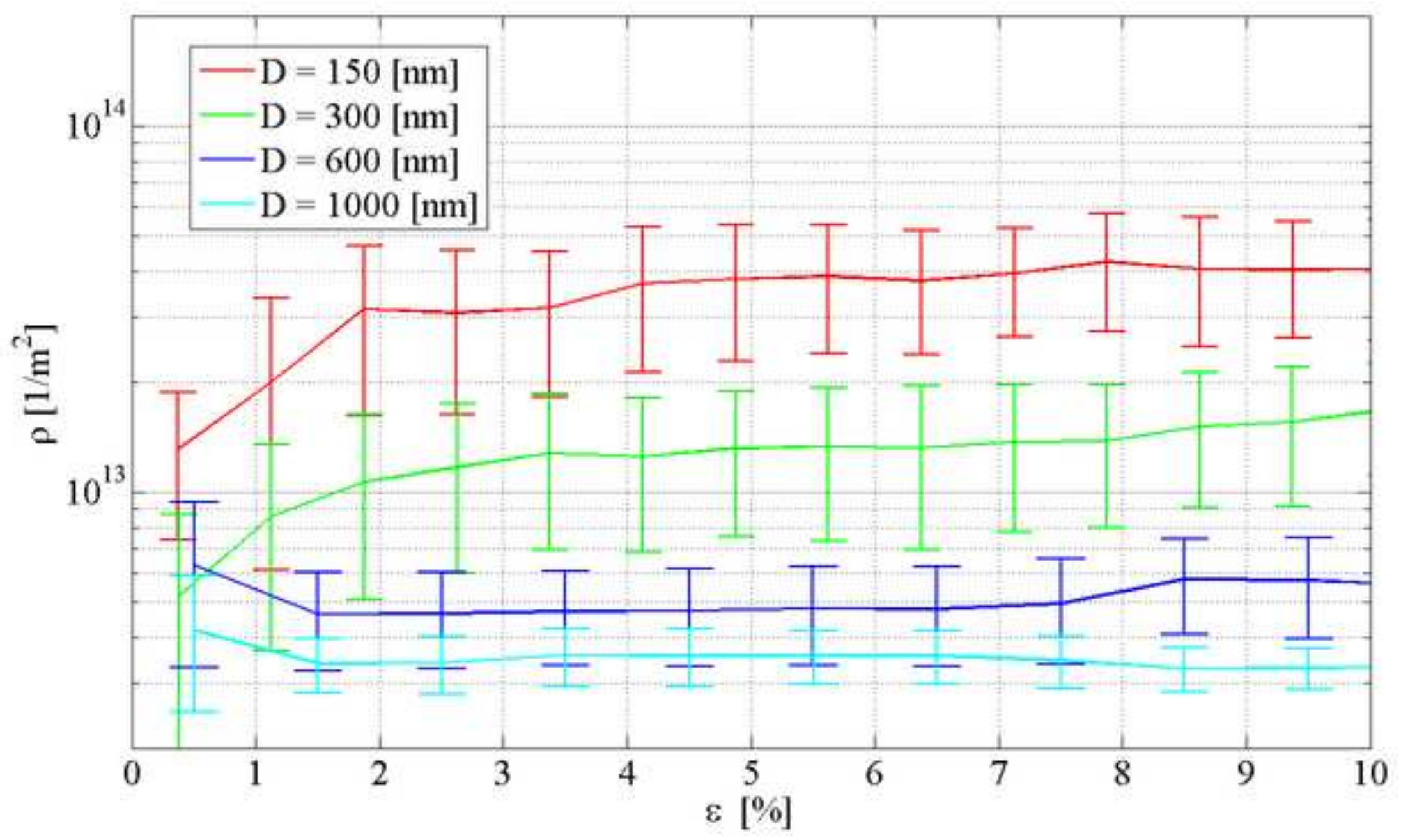




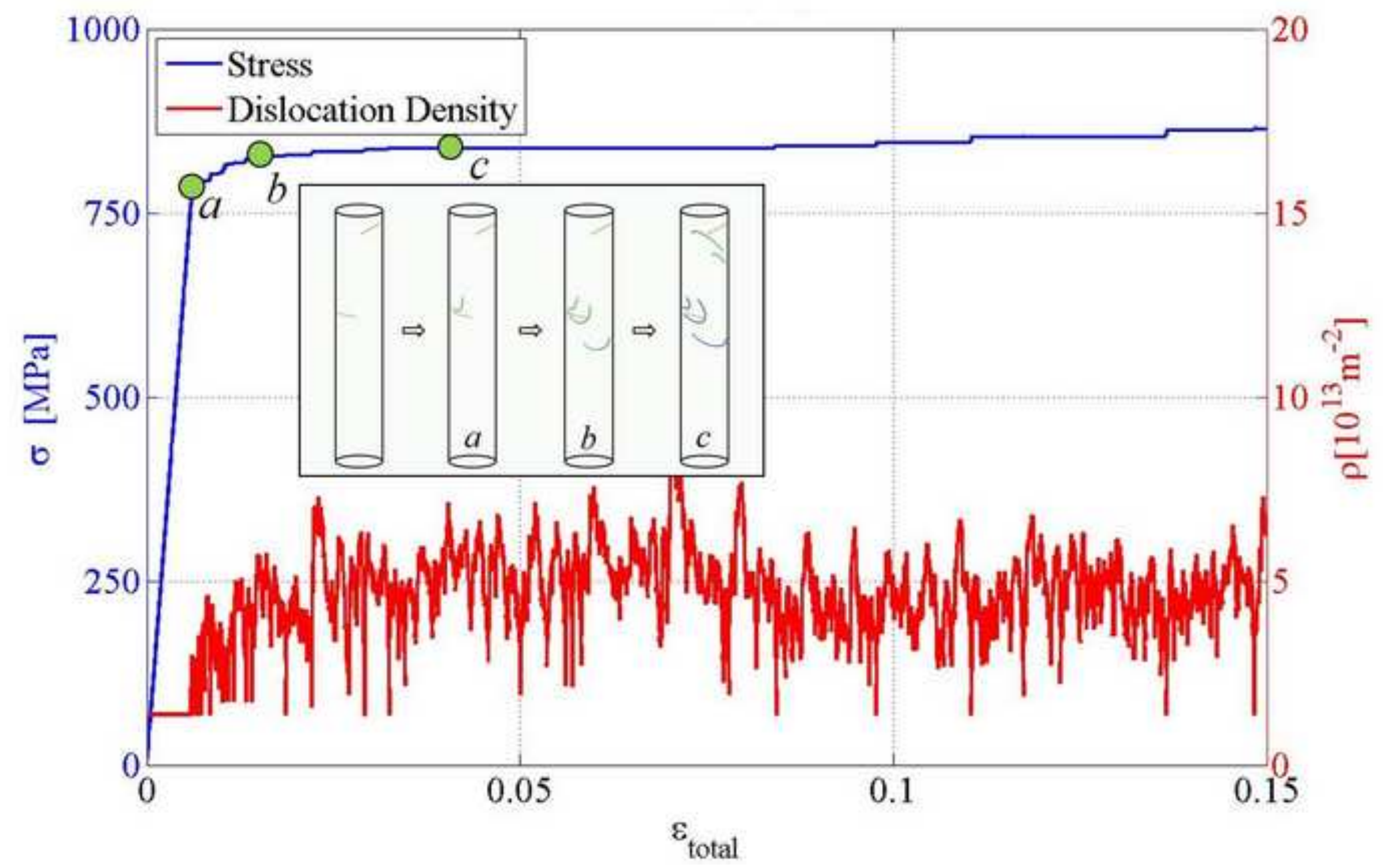




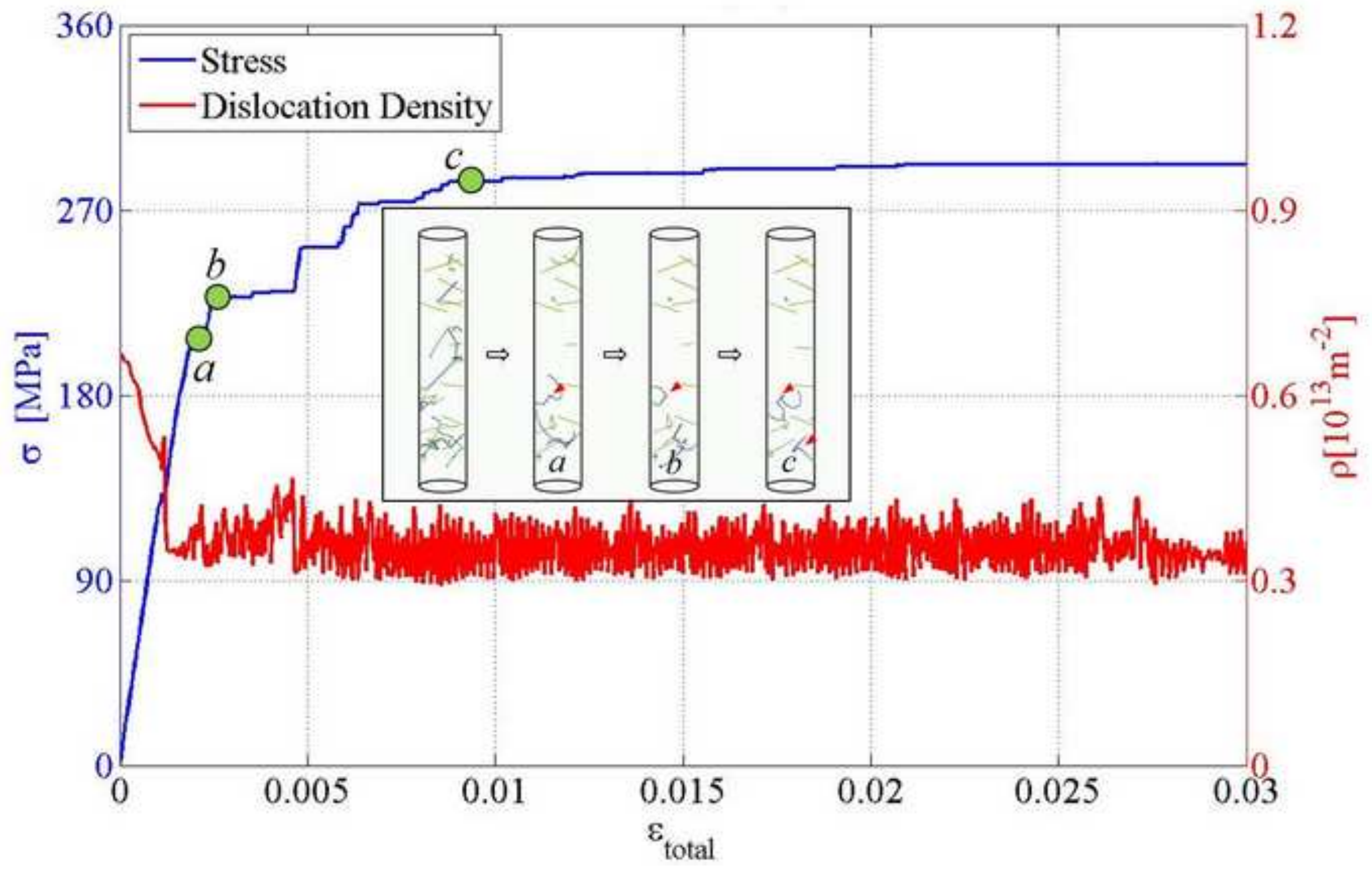




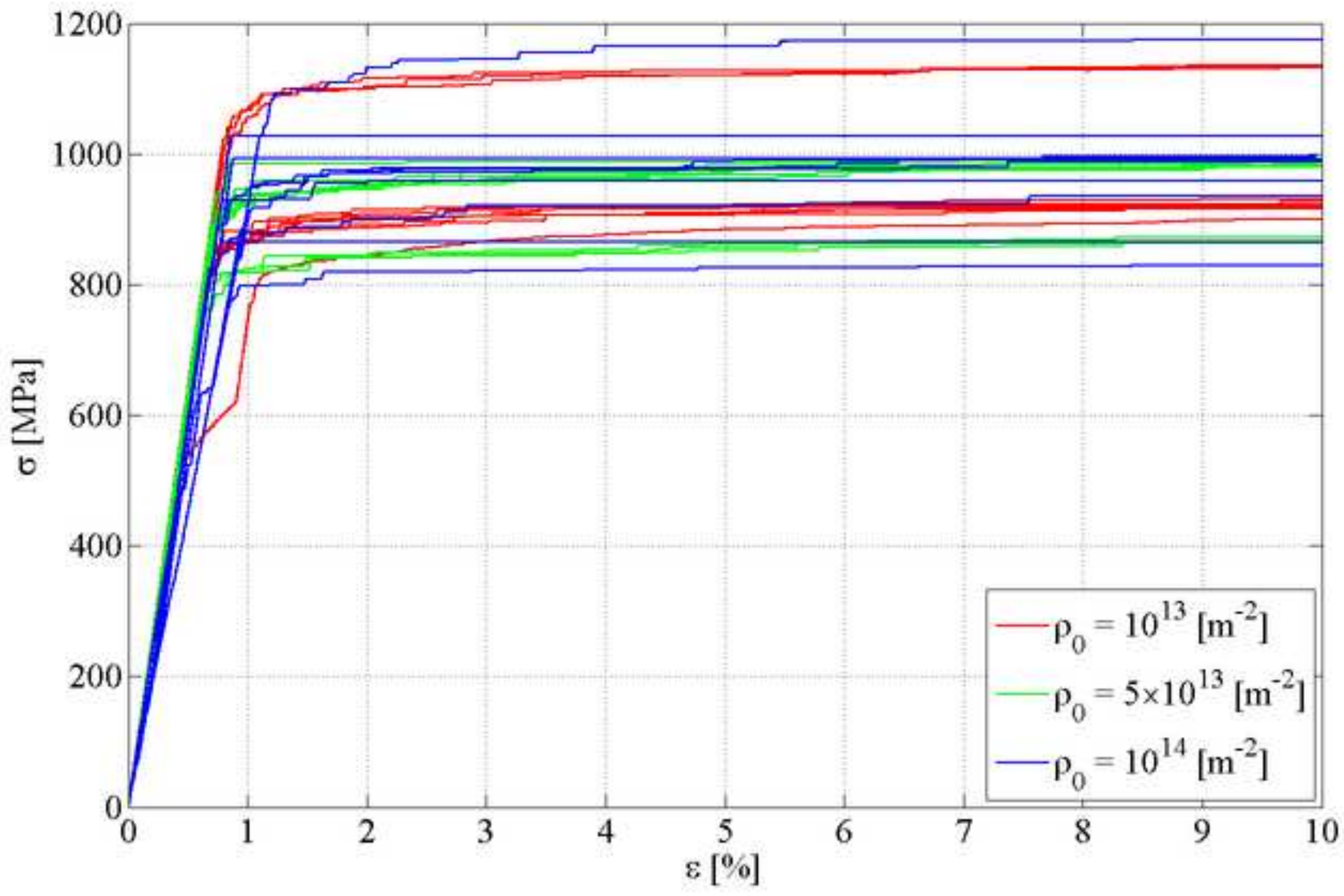




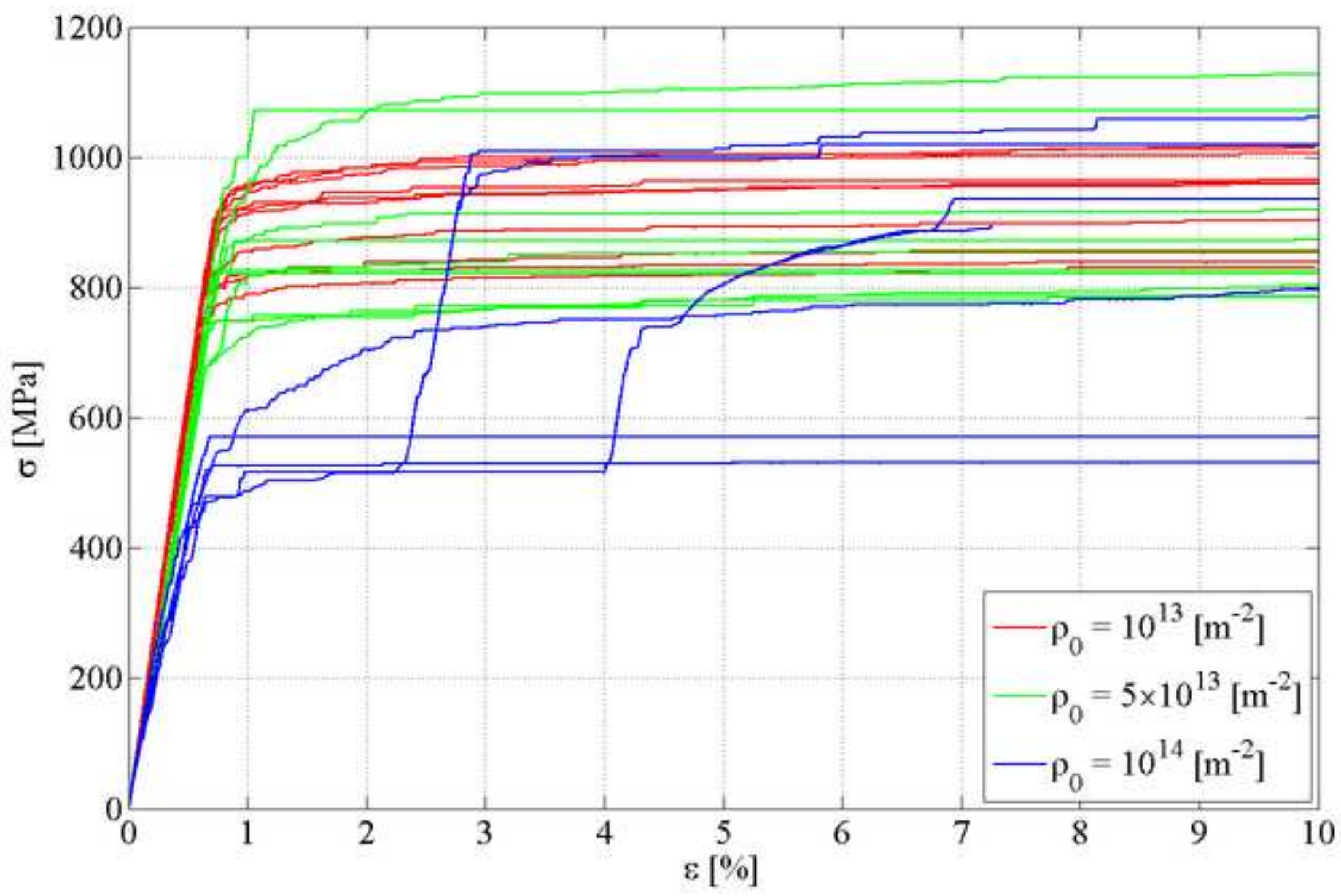




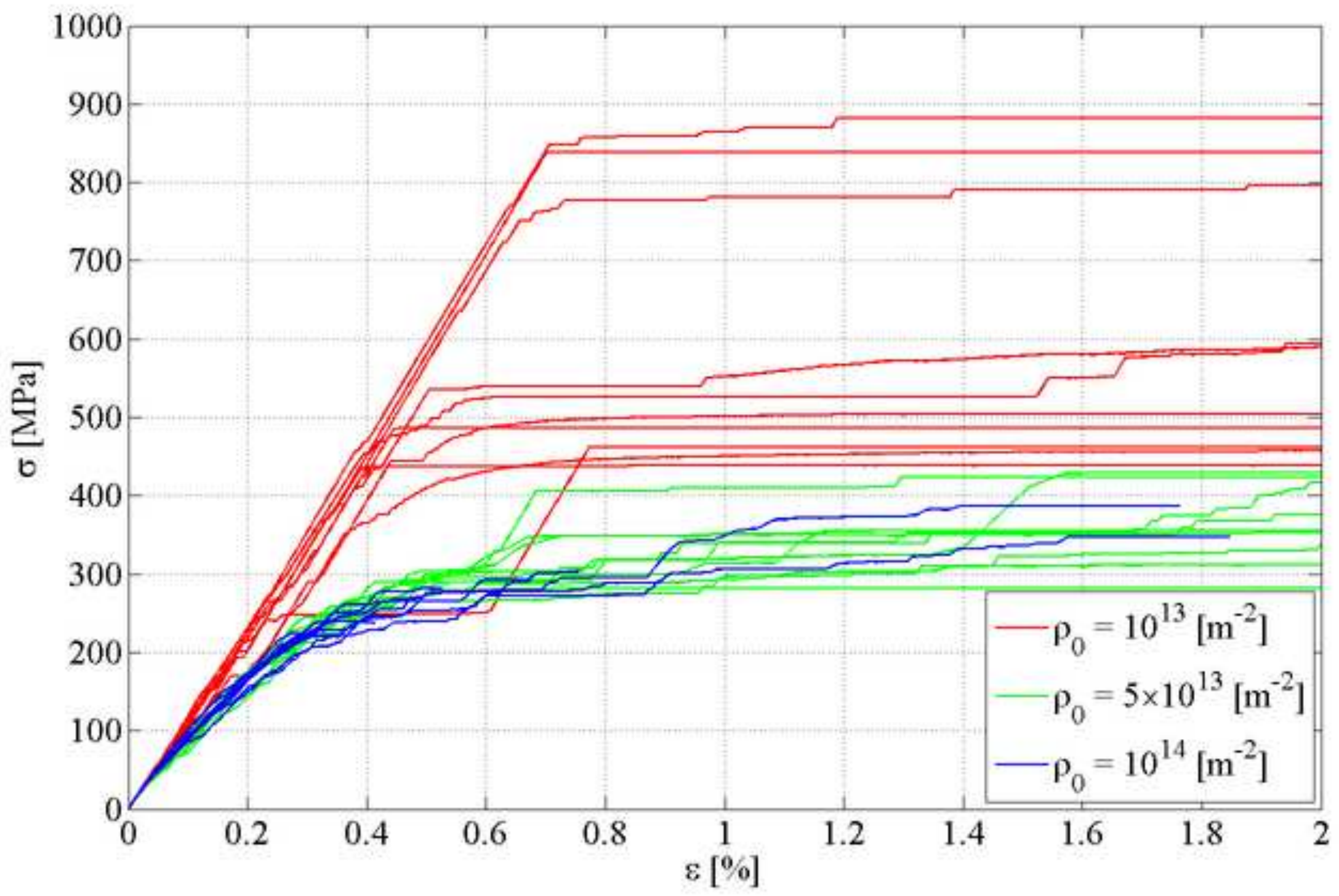




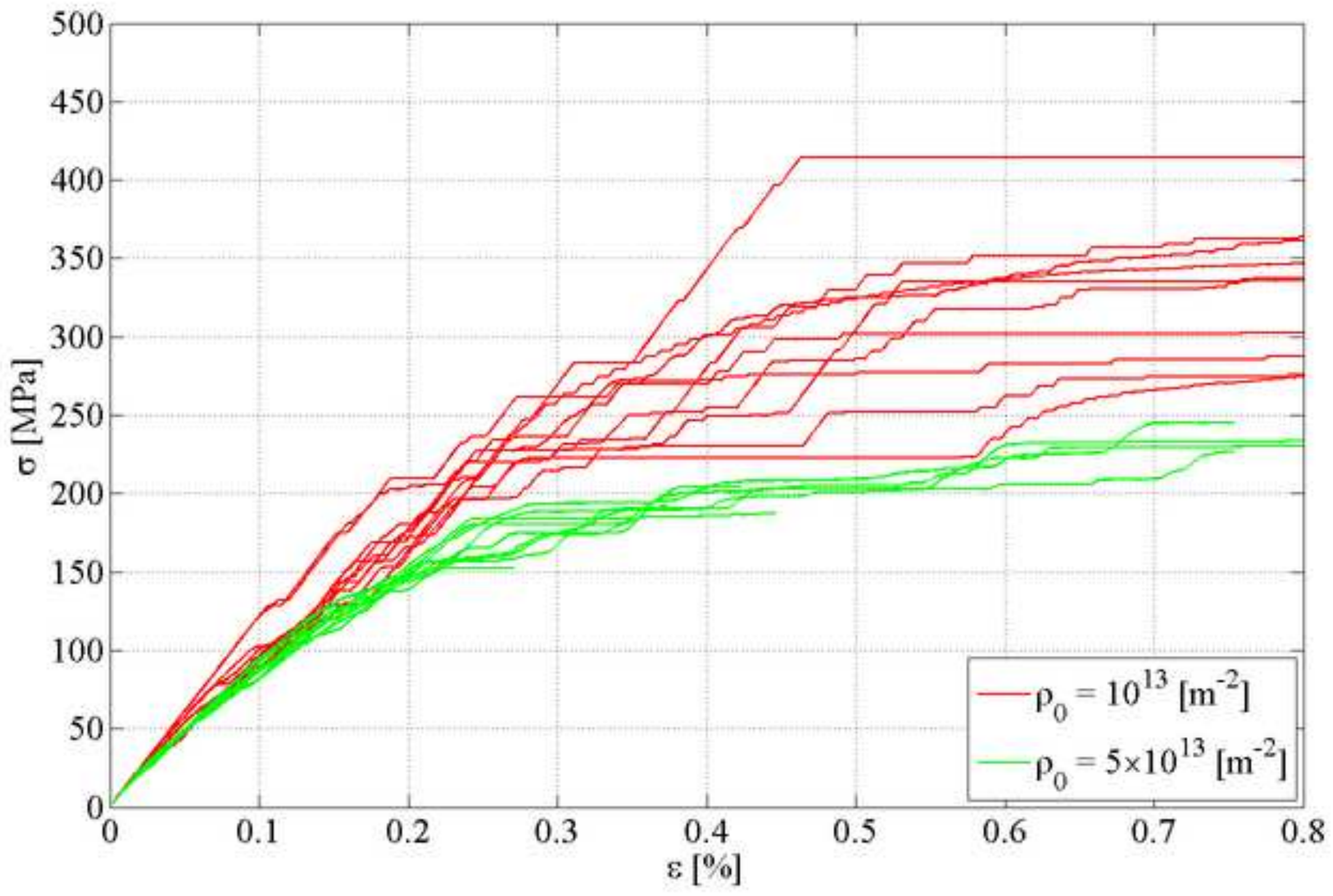




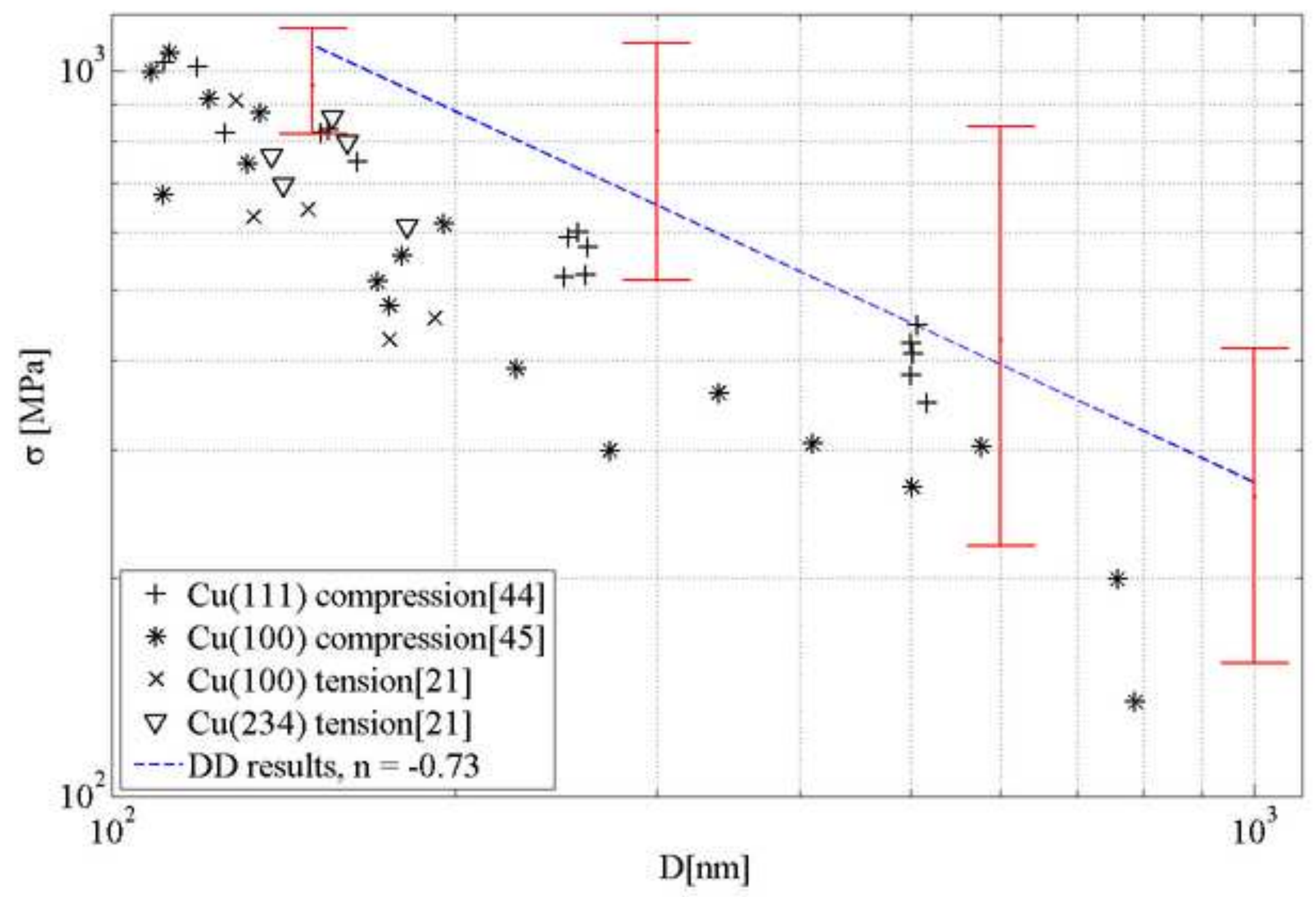




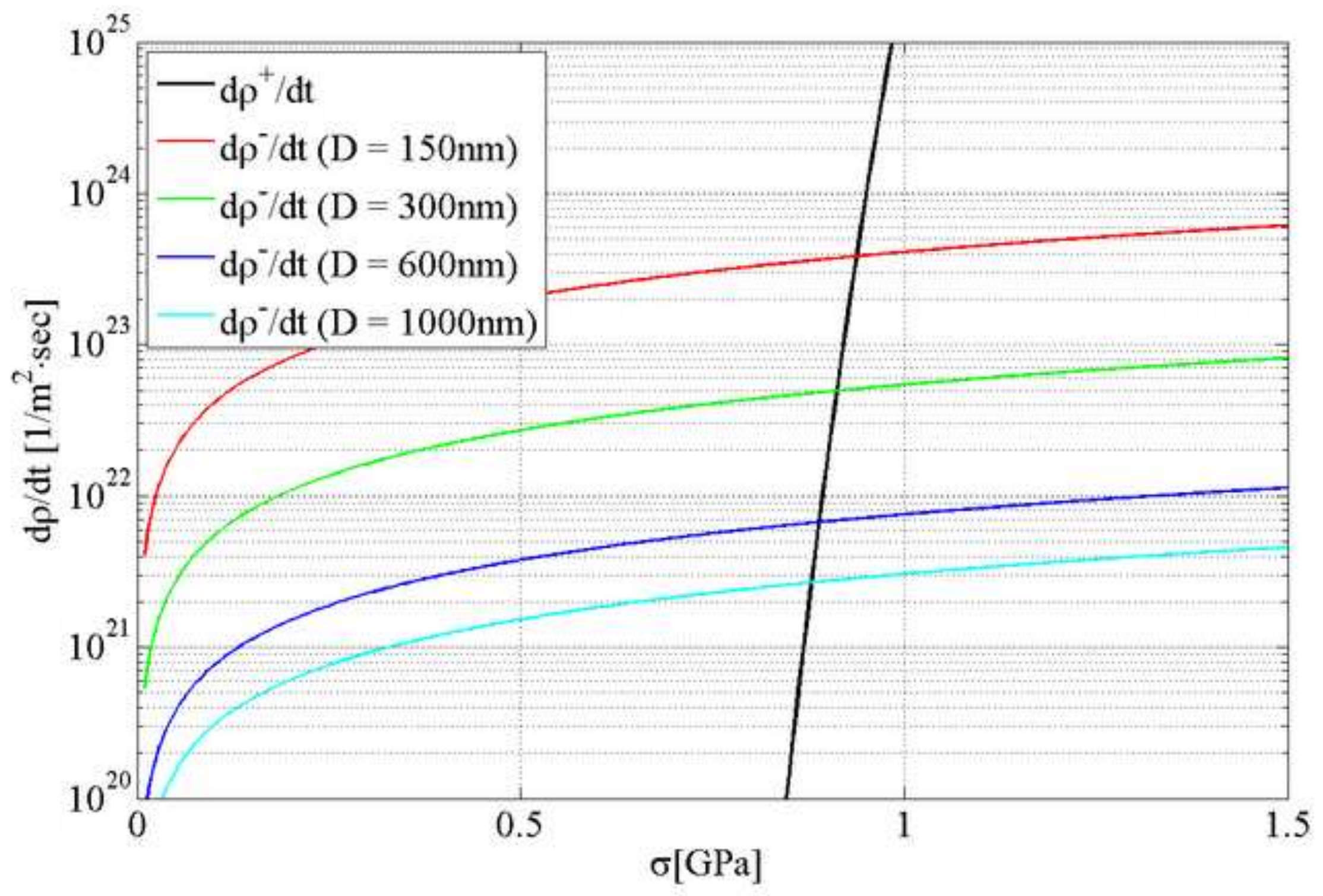




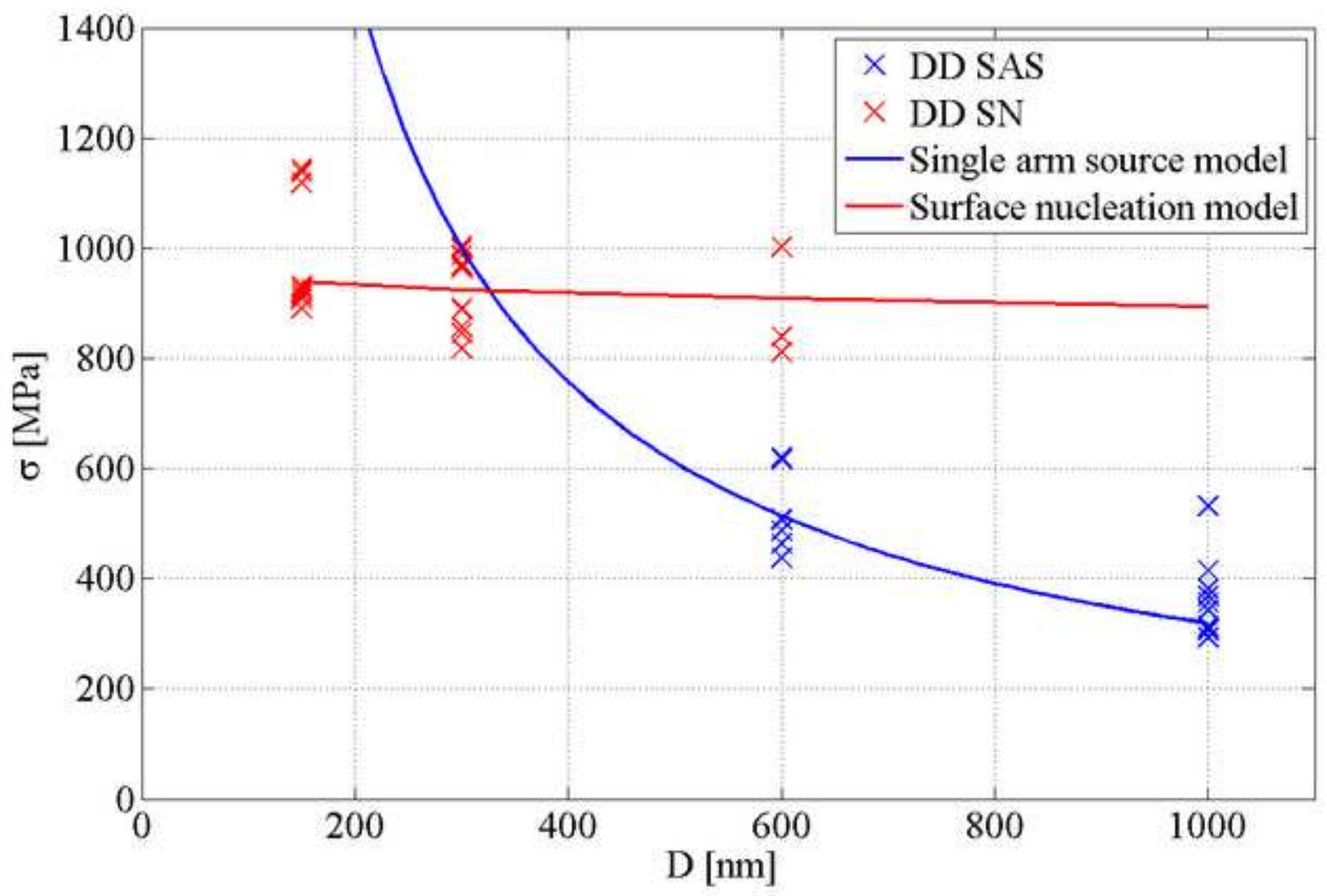




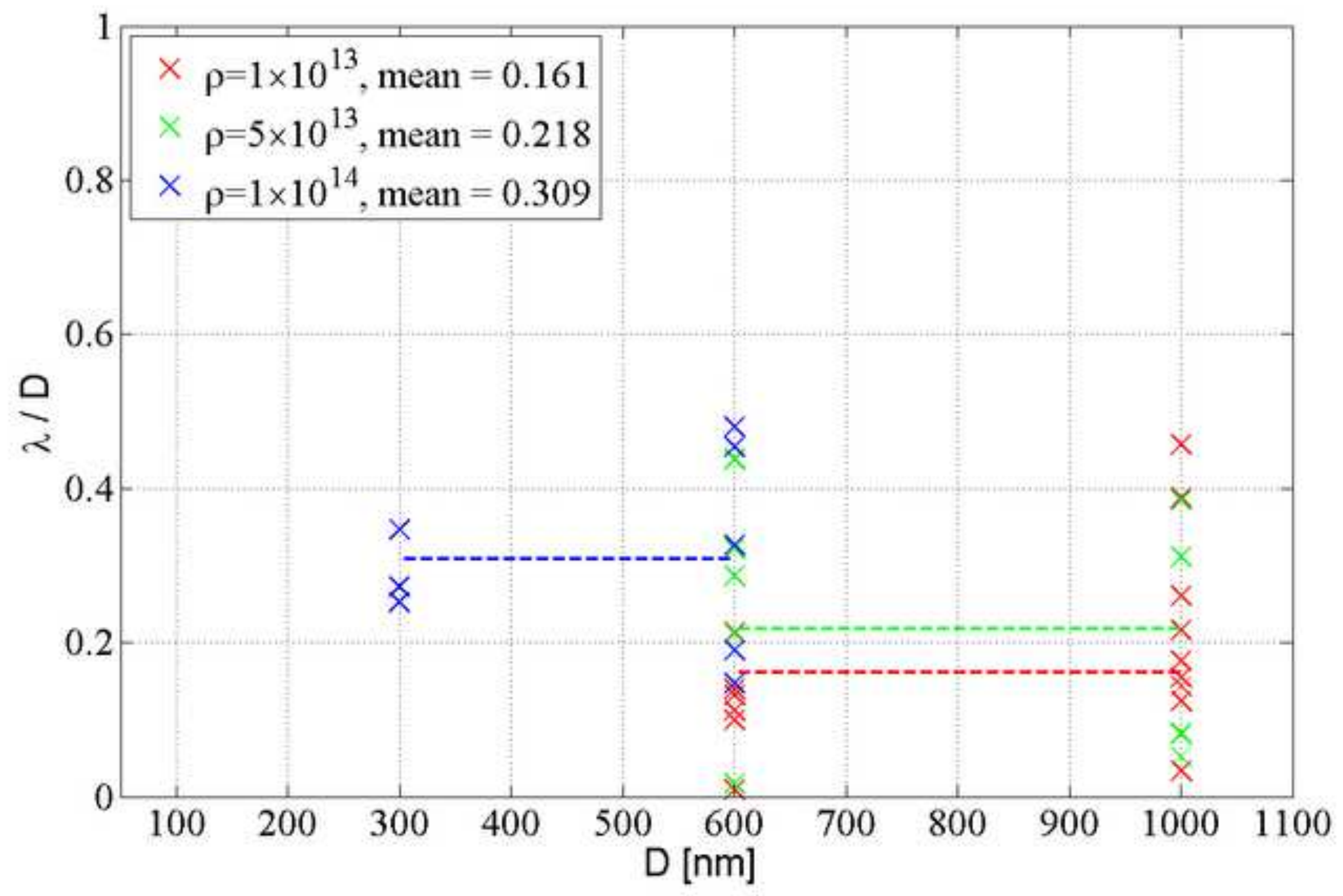




\section{Table captions}

Table 1. Material properties and operating parameters.

Table 2. Experimental conditions 
Table 1. Material properties and operating parameters.

\begin{tabular}{|c|c|c|}
\hline Material and parameters & Dimension & Value \\
\hline Shear modulus & {$[G P a]$} & 48 \\
\hline Poisson ratio & $\square$ & 0.34 \\
\hline Edge mobility & {$\left[\mathrm{Pa}^{-1} \mathrm{sec}^{-1}\right]$} & $10^{5}$ \\
\hline Screw mobility & {$\left[\mathrm{Pa}^{-1} \sec ^{-1}\right]$} & $10^{5}$ \\
\hline Burgers vector length $(b)$ & {$[m]$} & $2.556 \times 10^{-10}$ \\
\hline Temperature & {$[K]$} & 300 \\
\hline Attempt frequency $\left(v_{0}\right)$ & {$\left[\sec ^{-1}\right]$} & $1 \times 10^{13}$ \\
\hline Mean value of $\operatorname{SCF}(\bar{\alpha})$ & $\square$ & 1.0 \\
\hline Standard deviation $(\beta)$ in $\mathrm{SCF}$ & $\square$ & 1.0 \\
\hline Friction stress in $\mathrm{Cu}$ [61] & {$[M P a]$} & 34.6 \\
\hline
\end{tabular}


Table 2. Experimental condition.

\begin{tabular}{|c|c|c|c|c|c|c|c|}
\hline $\begin{array}{l}\text { Experiment } \\
\text { [Ref. No.] }\end{array}$ & $\begin{array}{c}\text { Crystal } \\
\text { Orientation }\end{array}$ & $\begin{array}{c}\text { Pillar } \\
\text { Fabrication }\end{array}$ & $\begin{array}{c}\text { Section } \\
\text { Geometry }\end{array}$ & $\begin{array}{l}\text { Diameter } \\
{[\mathrm{nm}]}\end{array}$ & $\begin{array}{l}\text { Strain rate } \\
{\left[10^{-3} / \mathrm{sec}\right]}\end{array}$ & $\begin{array}{c}\text { Size } \\
\text { Exponent }\end{array}$ & $\begin{array}{c}\text { Initial } \\
\text { Density }\left[m^{-2}\right]\end{array}$ \\
\hline Tension [21] & [001], [234] & FIB milling & Rectangle & $128 \sim 190$ & $\sim 5$ & $\cdot$ & $(5.6 \pm 0.3) \times 10^{14}$ \\
\hline Compression [44] & {$[111]$} & Electroplating & Rectangle & $100 \sim 500$ & $\sim 3$ & $0.63 \pm 036$ & $1.5 \times 10^{14}$ \\
\hline Compression [45] & {$[001]$} & FIB milling & Circle & $90 \sim 1700$ & $\sim 5$ & $0.89 \pm 0.20$ & $5 \times 10^{13}$ \\
\hline
\end{tabular}

